\title{
Serum gamma-glutamyltransferase and alkaline phosphatase in rheumatoid arthritis
}

\author{
RJ SPOONER, DH SMITH,* DOROTHY BEDFORD, PR BECK†
}

From the Centre for Rheumatic Disease and Department of Biochemistry, Royal Infirmary, Glasgow G4 OSF

SUMMARY Serum gamma-glutamyltransferase (GGT) and alkaline phosphatase (AP) were assayed in 98 consecutive patients with rheumatoid arthritis. Twenty-three patients had increased GGT activities and 45 an increased AP activity. Twelve patients showed an increase in both enzyme activities and AP isoenzyme studies were performed on seven of this group. In three subjects an increase in the bone isoenzyme was observed and in three others the increase in activity was attributed to the liver isoenzyme. The remaining patient, who probably suffered from coexistent primary biliary cirrhosis, showed an increase in both bone and liver isoenzymes. The liver involvement, suggested by the alkaline phosphatase isoenzyme results, was largely confirmed by the butanol extraction of GGT. The changes in these enzymes in this small series could not be related definitely to drug therapy.

It is concluded that though increases in GGT and AP are common in rheumatoid arthritis, this does not necessarily indicate hepatic involvement. Further isoenzyme studies are needed to define the extent to which organs other than the liver bring about increases in these serum enzymes in rheumatoid disease.

Previous studies in rheumatoid arthritis have shown biochemical abnormalities related to liver function in up to $6 \%$ of patients. ${ }^{12}$ More recently gammaglutamyltransferase (GGT, EC 2.3.2.2), which has been found to be a sensitive indicator of hepatic damage, ${ }^{3}$ has been used as an index of liver involvement in rheumatoid arthritis. ${ }^{4}$ However, some workers have suggested that increases in serum GGT may arise from organs other than the liver. ${ }^{6} 7$ Extraction of serum GGT with $n$-butanol has been found to be a useful means for distinguishing between increased activity due to liver disease and that due to other diseases. ${ }^{8}$ It was thought worthwhile therefore, to investigate the changes in GGT and alkaline phosphatase (AP, EC 3.1.1.1), using isoenzyme studies of AP and butanol extraction of GGT where appropriate, in an unselected group of patients with rheumatoid arthritis in an attempt to define more clearly the extent of hepatic involvement. The present paper describes the preliminary results of this investigation.

* Present address: Department of Rheumatology, Coventry and Warwickshire Hospital, Stoney Stanton Road, Coventry. $\dagger$ Present address: Department of Clinical Biochemistry, District General Hospital, Moorgate Road, Oakwood, Rotherham S60 2UD.

Accepted for publication 9 November 1981

\section{Patients and methods}

\section{PATIENTS}

Ninety-eight patients with rheumatoid arthritis seen consecutively at the Outpatient Department were studied. All patients satisfied the American Rheumatism Association diagnostic criteria for "definite" rheumatoid arthritis. ${ }^{9}$ No patient was excluded on the grounds of age, drug therapy or previous liver disease.

\section{METHODS}

For each patient an articular index of joint tenderness, ${ }^{10} x$-ray staging, ${ }^{11}$ and duration of arthritis were recorded. Rheumatoid factor was measured by a latex particleaggregation technique(William Warner, Hampshire). Gamma-glutamyltransferase was assayed at $37^{\circ} \mathrm{C}$ with a kinetic method using $\gamma^{-}$ glutamyl-3-carboxy-4-nitroanalide as substrate (BCL Lewes). Reference values for this laboratory are 1-35 U/1, no sex-related difference having been identified. Alkaline phosphatase was assayed at $37^{\circ} \mathrm{C}$ using the recommendations of the Scandinavian Society for Clinical Chemistry. ${ }^{12}$ Age and sex related reference values for this laboratory have been described. ${ }^{13}$ Alkaline phosphatase isoenzymes were separated on the basis of their heat stability using the techniques described by Moss and Whitby ${ }^{14}$ modi- 
fied by taking samples at five-minute intervals. Satisfactory resolution of defined clinical groups has been achieved. Extraction of GGT using $n$-butanol was performed as described by Beck. ${ }^{8}$ In the absence of hepatic disease greater than $75 \%$ of activity is retained post extraction. Other liver function tests (serum bilirubin, proteins, aspartate and alanine transaminases) were assayed using standard laboratory procedures.

Statistical analysis of the difference between groups was performed using the Mann Whitney test. Regression analysis was performed by the least squares method.

\section{Results}

Table 1 summarises the clinical and laboratory findings in the 98 patients ( 29 male, 79 female). Serum GGT activity was increased in 23 patients, only four of whom had activities greater than three times the upper reference limit, while AP activity was raised in 45 patients only one of whom showed a gross increase, all other values being below $900 \mathrm{U} / 1$.

Table 1 Clinical and laboratory data in 98 patients with rheumatoid arthritis

\begin{tabular}{lcc}
\hline Parameter & Median & Range \\
\hline Age (yr) & 55 & $22-72$ \\
Duration of arthritis (yr) & 8 & $0 \cdot 5-37$ \\
Articular index of joint tenderness & 12 & $0-48$ \\
Functional grade: Stage I & 1 & \\
(No of patients) $\quad$ II & 10 & \\
& 87 & \\
Rheumatoid factor (titre) & 0 & \\
ESR, Westergren (mm/h) & 64 & $0-2048$ \\
Albumin (g/l) & 32 & $4-128$ \\
Globulin (g/l) & 43 & $32-48$ \\
Total bilirubin ( $\mu$ mol/l) & 31 & $24-43$ \\
Alkaline phosphatase (U/l) & 6 & $3-18$ \\
Aspartate aminotransferase (U/l) & 270 & $119-1909$ \\
Gamma-glutamyltransferase (U/l) & 19 & $9-104$ \\
\end{tabular}

Twelve patients had increases in both serum enzyme activities and a significant correlation $(r=0.52$; $\mathrm{p}<0.01$ ) was found between them.

The results classified according to increased enzyme activity are given in Table 2 . Of the indices measured, ESR values were increased significantly, in the presence of raised AP activities. A weak correlation $(r=0.39 ; p<0.01)$ was found between ESR and AP. No association was observed between ESR and GGT or between articular index or rheumatoid factor and either enzyme.

The patient with the highest alkaline phosphatase (1909 U/l) and GGT (481 U/l) also had increased aspartate transaminase $(104 \mathrm{U} / \mathrm{l})$ and bilirubin (18 $\mu \mathrm{mol} / \mathrm{l})$. This woman had a markedly raised antimitochondrial antibody titre $(1 / 2048)$ and was probably suffering from primary biliary cirrhosis. Only two other patients had increased aspartate transaminase activities, and two had clinically enlarged livers.

Alkaline phosphatase isoenzyme studies and $n$-butanol extraction of GGT were performed on seven of the patients with increased activities of both these enzymes. Table 3 summarises the results. Three of the patients had an increase in the bone alkaline phosphatase fraction, while three had an increase in the liver fraction. Patient 2 (MM) had an increase in both fractions and is the patient described above as probably suffering also from primary biliary cirrhosis. The results of the extraction of GGT with $n$-butanol were supportive of a liver origin for three of the four patients with increases in liver alkaline phosphatase. The remaining patient (No 7) had only a slight increase in GGT with no evidence of liver involvement by $n$-butanol extraction.

\section{Discussion}

Liver involvement in rheumatoid arthritis is not

Table 2 Clinical and laboratory data in patients classified according to their AP and GGT values (median and range)

\begin{tabular}{|c|c|c|c|c|}
\hline & Normal & Increased $A P$ & Increased GGT & $\begin{array}{l}\text { Increased } \\
A P \text { and } G G T\end{array}$ \\
\hline Number & 40 & 33 & 11 & 12 \\
\hline \multirow[t]{2}{*}{$\operatorname{AP}(U / 1)$} & 225 & 336 & 251 & 362 \\
\hline & $(119-274)$ & $(282-598)$ & $(158-286)$ & $(305 \cdot 1909)$ \\
\hline GGT (U/1) & 15 & 20 & 47 & 84 \\
\hline & $(10-35)$ & $(10-33)$ & $(36-81)$ & $(42-481)$ \\
\hline Articular index of joint tenderness & 15 & 12 & 6 & 12 \\
\hline & $(0-46)$ & $(0-48)$ & $(0-12)$ & $(0-18)$ \\
\hline ESR, Westergren (mm/h) & 24 & $45 * *$ & 27 & $58 *$ \\
\hline & $(4-105)$ & $(4-121)$ & (6-67) & $(5-128)$ \\
\hline Rheumatoid factor & 64 & 64 & 128 & 64 \\
\hline (titre) & $(0-2048)$ & $(0-2048)$ & $(16-1024)$ & $(0-512)$ \\
\hline
\end{tabular}

Asterisks indicate a significant difference from the normal group-

$-p<0.05$.

$* * \mathrm{p}<0.01$. 
Table 3 Alkaline phosphatase isoenzymes and n-butanol extraction of GGT in patients with rheumatoid arthritis

\begin{tabular}{|c|c|c|c|c|c|}
\hline \multirow[t]{2}{*}{ Patients } & \multirow{2}{*}{$\begin{array}{l}\text { Serum } G G T \\
(U / l)\end{array}$} & \multirow{2}{*}{$\begin{array}{l}\text { GGT activity remaining after } \\
\text { butanol extraction } *(\%)\end{array}$} & \multirow{2}{*}{$\begin{array}{l}\text { Serum } A P \\
(U / I)\end{array}$} & \multicolumn{2}{|l|}{$A P$ isoenzymes } \\
\hline & & & & Ratio liver: bone $\dagger$ & Source \\
\hline 1 & 382 & 88 & 362 & $3: 1$ & Bone \\
\hline 2 & 481 & 74 & 1909 & $3: 1$ & Liver + bone \\
\hline 3 & 76 & 70 & 503 & $9: 1$ & Liver \\
\hline 4 & 98 & 88 & 363 & $1 \cdot 6: 1$ & Bone \\
\hline 5 & 110 & 68 & 873 & $8: 1$ & Liver \\
\hline 6 & 115 & 65 & 307 & $1: 1$ & Bone \\
\hline 7 & 46 & 82 & 586 & $5: 1$ & Liver \\
\hline
\end{tabular}

Reference values: ${ }^{*}$ Normal $=>75 \%$; supportive of liver source of GGT $<75 \%$. + Normal $=2: 1$.

uncommon. Serum alkaline phosphatase and GGT have been found to be increased in rheumatoid arthritis, and this has been suggested as being evidence for liver involvement in rheumatoid disease. ${ }^{4}{ }^{5}$ Lowe et $\mathrm{l}^{5}$ were able to relate increases in GGT to other indices of disease activity-for example, ESR and plasma viscosity. In the present study no statistical difference was noted between the levels of ESR, articular index and rheumatoid factor in patients with normal compared to those with increased GGT. However there is a definite, though weak, association between AP and ESR. This may reflect a non-specific response to the inflammatory process. It is important to note that though the patients reported by Lowe $e$ al did show increases in aspartate and alanine transaminases, they were under therapy with salicylates which may themselves be hepatotoxic, particularly in the high doses used in treating rheumatoid disease. ${ }^{15}$ Other workers have also suggested that the increases in GGT and alkaline phosphatase support liver involvement by the rheumatoid process. However, organ specific separation has not been generally performed.

In the present unselected series of patients increases of serum alkaline phosphatase occurred in $45 \%$ of patients, while GGT was increased in $23 \%$. Alkaline phosphatase isoenzymes were performed in seven of the patients in whom both alkaline phosphatase and GGT were increased. In three cases the liver isoenzyme was raised and in three the bone isoenzyme predominated. In the last case both isoenzymes were raised, and this patient has a probable diagnosis of primary biliary cirrhosis. The association between primary biliary cirrhosis and rheumatoid disease is well recognised. ${ }^{16}$

Extraction of serum GGT with $n$-butanol has been found to be capable of distinguishing between patients with liver disease, and those in whom other causes of increased GGT are implicated. ${ }^{8}$ Its use in the present studies has confirmed the alkaline phosphatase isoenzymes studies. In those patients in whom liver involvement has not been $\mathrm{e}_{\mathrm{c}}^{\mathrm{j}}$ identified by alkaline phosphatase isoenzymes or by butanol extraction of GGT the source of the increase in GGT is unknown, although it may be related to the inflammatory or catabolic process involved in rheumatoid disease. ${ }^{17}$ However, it has not been possible to show a relation between increased GGT and indices of rheumatoid disease activity. In future studies, liver function will need to be assessed using a variety of other parameters-for example, serum bile acids, as well as those used here.

In the present series of unselected cases no attempt was made to exclude specific drug therapy. Thus it is possible that some of the changes identified were related to therapy. Six patients with increases in serum GGT were receiving gold injections, whilst a further six were being treated with indomethacin. Both of these drugs have been reported to cause liver damage. ${ }^{1819}$ The question as to the relative contributions of drug therapy and disease to the biochemical changes in rheumatoid arthritis can be resolved only by further prospective studies of patients before and after therapy. Our results do suggest, however, that further tissue specific isoenzyme studies are necessary to define the extent to which liver involvement is responsible for increases in alkaline phosphatase and GGT activities in rheumatoid arthritis.

\section{References}

${ }^{1}$ Cockel R, Kendall MJ, Becker JF, Hawkins CF. Serum biochemical values in rheumatoid disease. Ann Rheum Dis 1971;30:166-70.

2 Webb J, Whaley K, MacSween NM, Nuki G, Carson DW, Buchanan WA. Liver disease in rheumatoid arthritis and Sjögren's syndrome: prospective study using biochemical and serological markers of hepatic dysfunction. Ann Rheum Dis 1975;34:70-81.

${ }^{3}$ Goldberg DM, Martin JV. The role of gamma-glutamyltranspeptidase activity in the diagnosis of hepatobiliary disease. Digestion 1975;12:232-46.

${ }^{4} \mathrm{Rau}$ R. Gamma-glutamyltranspeptidase in rheumatoid arthritis. $Z$ Rheumatol 1977;36:49-59.

${ }^{5}$ Lowe JR, Pickup ME, Dixon JS, et al. Gamma-glutamyl- 
transpeptidase levels in arthritis: a correlation with clinical and laboratory indices of disease activity. Ann Rheum Dis 1978;37:428-31.

${ }^{6}$ Agostoni A, Ideo G, Stabilini R. Serum gamma-glutamyltranspeptidase activity in myocardial infarction. $\mathrm{Br}$ Heart J 1965;27:688-90.

7 Beck PR. PhD thesis. University of Glasgow, 1978.

${ }^{8}$ Beck PR. Butanol extraction of serum and urinary gammaglutamyltransferase and its application in clinical diagnosis. Ann Clin Biochem 1978;15:151-6.

${ }^{9}$ Ropes MW, Bennett GA, Cobb S, Jacox R, Jessar RA. 1958 revision of diagnostic criteria for rheumatoid arthritis. Arthritis Rheum 1959;2:16-20.

${ }^{10}$ Ritchie DM, Boyle JA, McInnes JM, et al. Clinical studies with an articular index for the assessment of joint tenderness in patients with rheumatoid arthritis. $Q J$ Med 1968;37:393-406.

11 Steinbroker O, Traeger CH, Batterman RC. Therapeutic criteria in rheumatoid arthritis. JAMA 1949;140: 659-62.

12 Committee on Enzymes of the Scandinavian Society for Clinical Chemistry and Clinical Physiology. Recommended methods for the determination of four enzymes in blood. Scand J Clin Lab Invest 1974;33: 291-306.

${ }^{13}$ Gardner MD, Scott R. Frequency distribution and "reference values" of plasma alkaline phosphatase (EC 3.1.3.1) activity in the adult population of a Scottish new town. J Clin Pathol 1978;31:1202-6.

${ }^{14}$ Moss DW, Whitby LG. A simplified heat-inactivation method for investigating alkaline phosphatase isoenzymes in serum. Clin Chim Acta 1975;61:63-71.

15 Bryant CE. Salicylate-induced hepatotoxicity: a review. J Okla State Med Assoc 1978;71:284-7.

${ }^{16}$ Sherlock S, Scheuer PJ. Presentation and diagnosis of 100 patients with primary biliary cirrhosis. $N$ Engl $J$ Med 1973;289:674-8.

${ }^{17}$ Kataja J, Gordin R. Serum gamma-glutamyltranspeptidase and alkaline phosphatase in severely injured patients. Acta Chir Scand 1970;136:277-81.

18 Fenech FF, Bannister WH, Greck JL. Hepatitis with biliverdinaemia in association with indomethacin therapy. Br MedJ 1963 ;iii:155-6.

19 Schenker S, Olson KN, Dunn D, Breen KJ, Combes B. Intrahepatic cholestasis due to therapy of rheumatoid arthritis. Gastroenterology 1973;64:622-9.

Requests for reprints to: Mr RJ Spooner, Department of Biochemistry, Royal Infirmary, Glasgow G4 OSF, Scotland. 